\title{
INTERPRETIVE SUMMARIES, NOVEMBER 2011
}

Invited review: Dairy intake and bone health: A viewpoint from the state of the art. By Caroli et al., page 5249. The complex relationships between intake of milk and dairy products and bone health are examined, with particular emphasis on osteoporosis. An adequate calcium intake during growth has long been recognized to be the most critical nutritional factor in achieving optimal peak bone mass. The greatest amount of dietary calcium is obtained from milk and dairy foods. We review the literature on favorable effects of milk and dairy products on bone health. Discordant data, mainly on the risk of fractures, are discussed.

Growth of Lactobacillus paracasei ATCC 334 in a cheese model system: A biochemical approach. By Budinich et al., page 5263. Nonstarter lactic acid bacteria (NSLAB) such as Lactobacillus paracasei grow to high levels in ripening cheese and can have a significant effect (positive or negative) on flavor development. The dairy industry is interested in methods to control the NSLAB microbiota, but those efforts are limited by a lack of understanding of the factors that influence NSLAB growth in cheese. We used a medium prepared from ripening Cheddar cheese as a model to explore $L b$. paracasei growth, substrate utilization, and metabolic product formation in cheese. Results provide valuable new information on the biochemistry and physiology of $L b$. paracasei during growth in ripening cheese.

Visible and near-infrared spectroscopic analysis of raw milk for cow health monitoring: Reflectance or transmittance? By Aernouts et al., page 5315. The composition of the produced milk provides valuable information on the cow's metabolism and health. In this study, we investigated the potential of two visible/near-infrared spectroscopic measurement modes (reflectance and transmittance) and different wavelength ranges for on-line prediction of the major components in raw milk, and thus individual cow health monitoring.

Effect of storage and separation of milk at udder quarter level on milk composition, proteolysis, and coagulation properties in relation to somatic cell count. By Forsbäck et al., page 5341. A major problem in dairy production is udder health (i.e., mastitis), which causes deteriorated milk quality. Another factor that affects milk quality is storage of milk, which is common in practice. This study evaluated the effect of mastitis and storage on milk quality, especially milk composition and coagulation properties. Both storage and udder health affect milk quality. Separation of milk from udder quarters with impaired udder health will result in improved milk quality at the cow level.
Animal performance and milk fatty acid profile of dairy goats fed diets with different unsaturated plant oils. By Martínez Marín et al., page 5359. Adding high oleic oil (HOSFO), regular sunflower oil (RSFO), or linseed oil (LO) to dairy goat diets moderately changed production performance, but substantially modified the fatty acid profile of milk fat. Vaccenic acid content was increased more than 3.5-fold with RSFO and LO diets, and doubled with the HOSFO diet compared with the control diet. Rumenic acid was nearly tripled with RSFO and LO diets, whereas trans-10-18:1 was higher with HOSFO and RSFO diets. The LO treatment decreased the n-6:n-3 fatty acid ratio, and RSFO increased it. Addition of LO to dairy goat diets seems the best choice from a human health perspective.

Short communication: Effect of subclinical mastitis on proteolysis in ovine milk. By Martí-de Olives et al., page 5369. Mammary infection entails an increase of proteolytic enzymes in milk, which can degrade the quality of the proteins, leading to a decrease in the technological suitability of milk. We studied the effect of intramammary infection (IMI) on different ewe milk proteolysis products by a direct comparison between infected and uninfected glands. The presence of a subclinical IMI involved an increase of the products of casein hydrolysis: the protease-peptone fraction and minor $(\mathrm{m})$ caseins as well as a decrease of $\beta$-casein in ewe milk.

Short communication: Suitability of fluorescence spectroscopy for characterization of commercial milk of different composition and origin. By Ntakatsane et al., page 5375. Maintaining consistent and high food quality has been a challenge to food producers and processors. This study applied a rapid analytical technique based on front-face fluorescence spectroscopy (FFFS) for characterizing commercial milks of different composition manufactured in various countries. Pasteurized and ultra-high temperature milks with different compositions showed characteristic patterns, and international and domestic brands were discriminated. This study demonstrates the ability and sensitivity of FFFS to rapidly discriminate and classify commercial milk with various compositions and processing conditions.

Covariance among milking frequency, milk yield, and milk composition from automatically milked cows. By Løvendahl and Chagunda et al., page 5381. Automatic milking allow cows to access milking units at greatly varying time intervals. A large part of this variation is caused by differences between cows as 
individuals. Individual differences clearly exist in many traits such as milk yield and composition and milking frequency. We found that cows with high yield went to milking more often but had less fat in their milk compared with cows with lower yield. Individual differences between cows persisted throughout their lactation. The findings were based on data from a single experimental herd of Holstein, Red Dane, and Jersey cows and relationships were only estimated within breed.

Feasibility assessment of a tunnel-ventilated, water-padded barn on alleviation of heat stress for lactating Holstein Cows in a humid area. By Shiao et al., page 5393. In Taiwan, persistently hot and humid weather seriously restricts cow welfare and milk production. Heat stress is most directly and effectively improved by improving the barn environment. In this study, we evaluated the contributions of a tunnelventilated and water-padded barn to reducing cow heat stress. We found that increasing air speed and using sprinkler cooling benefited the cows and improved their lactation performance. This modified barn environment was more comfortable for lactating Holstein cows.

Lipopolysaccharide and lipoteichoic acid induce different immune responses in the bovine mammary gland. By Wellnitz et al., page 5405. This study compared the immune response of the bovine mammary gland to Escherichia coli lipopolysaccharide (LPS) and Staphylococcus aureus lipoteichoic acid (LTA) in vivo. A dosage of LPS and LTA was chosen that resulted in an increase of somatic cell count to a similar maximum. However, the induction of important immune factors differed between LPS and LTA treatment in milk and milk cells. Differences in the course of mastitis resulting from E. coli and Staph. aureus infections shown in practice may be partly explained by these different responses to LPS and LTA.

Phenotypic effects of calving ease on the subsequent fertility and milk production of dam and calf in UK Holstein-Friesian heifers. By Eaglen et al., page 5413. Calving is a key event for the dairy cow, with calving ease being an economically significant nonproduction trait for the dairy cattle industry. Considering the complexity of the parturition process, it is highly plausible that a difficult calving will affect the subsequent performance of both of the animals involved. Our results show clear detrimental effects of a difficult calving on the subsequent reproductive performance of the dam. Furthermore, we show that a difficult calving impairs the subsequent milk production of both the dam and the adult calf. To estimate lactation curves by the fitting of cubic splines allowed the determination of scale and size of losses in milk yield during the lactation following different degrees of calving ease. The detection of a long-term effect of calving ease on the milk production of the adult calf is a new finding to the dairy cattle industry.

A simple method to score digital dermatitis in dairy cows in the milking parlor. By Relun et al., page 5424. Digital dermatitis is a major cause of lameness in cows worldwide. The most commonly used diagnostic test is foot inspection in a trimming chute, but this is a labor-intensive procedure. This study assessed the performance of an inexpensive and simple method, based on a swiveling mirror and a powerful headlamp, which can be used in the milking parlor to score digital dermatitis. The method demonstrated both accuracy and good agreement within and between observers, and thus could be used by researchers to evaluate the effectiveness of digital dermatitis control measures.

Induced hypoglycemia for 48 hours indicates differential glucose and insulin effects on liver metabolism in dairy cows. By Kreipe et al., page 5435. Effects of an induced hypoglycemia over $48 \mathrm{~h}$ on metabolic parameters in plasma and liver of midlactation cows were studied. Three treatments were used, including a hyperinsulinemic hypoglycemic clamp (HypoG, $\mathrm{n}=6$ ) to obtain a glucose concentration of 2.5 $\mathrm{mmol} / \mathrm{L}$, a hyperinsulinemic euglycemic clamp (EuG, $\mathrm{n}=6)$, and a control treatment $(\mathrm{NaCl}, \mathrm{n}=6)$. Milk yield decreased in HypoG and liver mitochondrialphosphoenolpyruvate-carboxykinase mRNA abundance was upregulated. The EuG treatment decreased plasma $\beta$-hydroxybutyrate. Treatment differences for genes related to lipid metabolism were absent. Metabolic regulatory events are directed, apart from hormones, by the level of metabolites, either in excess (e.g., free fatty acids) or in shortage (e.g., glucose).

The effect of lameness prevalence on dairy farm level technical efficiency: An adjusted data envelopment analysis approach. By Barnes et al., page 5449. Western agricultural policy seeks to engender sustainable economic growth. This requires measures to be developed that integrate food production and the sometimes conflicting goals of improving animal welfare and environmental management. This paper developed a measure of technical efficiency with lameness prevalence for a number of UK farms and found that active management for lameness helped to improve technical efficiency measures. Our data have implications for the mix of resources on the farm but also for society in terms of understanding the requirements of sustainable growth.

Alteration of the nutrient uptake by the udder over an extended milking interval in dairy cows. By Guinard-Flament et al., page 5458. Decreasing milking frequency can strongly impair the milk yield of dairy cows and offers a useful model for gaining deeper 
insights into the mammary utilization of nutrients circulating in blood plasma for milk synthesis. A trial was carried out to describe the mammary nutritional adjustments linked to the downregulation of milk synthesis as milk accumulated over $36 \mathrm{~h}$ in the udder.

Animal welfare in cross-ventilated, compost bedded pack, and naturally ventilated dairy barns in the upper Midwest. By Lobeck et al., page 5469. Observational data were collected from 18 dairy farms in Minnesota and South Dakota to evaluate animal welfare in 3 types of housing systems using outcomebased measurements (locomotion, hock lesions, body condition, hygiene, respiration rates, mortality, and mastitis prevalence). Dairy cattle housed in compostbedded pack barns had reduced prevalence of lameness and hock lesions compared with those housed in crossventilated and naturally ventilated freestall barns and no adverse associations with body condition, respiration rates, or mastitis prevalence. Comparing the two freestall housing options, cross-ventilated barns had greater cow comfort indices than naturally ventilated barns.

Temporal feed restriction and overstocking increase competition for feed by dairy cattle. By Collings et al., page 5480. Reduced availability of resources, in time or space, can increase competition for these resources. This study tested the effects of overstocking at the feed bunk and restricting feed access time on the behavior of lactating Holstein cows. Overstocking and reduced feed access time increased competitive behavior at the feed bunk and reduced the time that cows were able to spend feeding. These behavioral responses were greatest when the effects of overstocking and reduced access time were combined.

The effect of lameness on the fertility of dairy cattle in a seasonally breeding pasture-based system. By Alawneh et al., page 548\%. Lameness is a cause of significant economic loss; one key way that it causes such loss is by reducing fertility. The effect of lameness on fertility may be different for cattle that are bred seasonally and pasture-fed compared with nonseasonally calving, housed cattle. In this study of lameness in a 463-cow dairy herd, lame cows had a daily probability of conceiving to artifical insemination 0.78 times that of nonlame cows. In addition, lame cows took $12 \mathrm{~d}$ longer to conceive compared with nonlame cows.

The use of dermal fibroblasts as a predictive tool of the toll-like receptor 4 response pathway and its development in Holstein heifers. By Green et al., page 5502. The innate immune response potential of heifers was analyzed using dermal fibroblast cultures established from 15 animals when they were
5, 11, and 16 mo of age. Production of interleukin- 8 by the fibroblasts in response to lipopolysaccharide (LPS) revealed substantial between-animal variation. The relative ranking of heifers remained consistent with age, although an increased responsiveness was seen in fibroblasts from older animals. Four low- and four highresponsive heifers were also challenged with intravenous LPS in vivo. High-responsive heifers exhibited higher plasma levels of the cytokines interleukin- 8 and tumor necrosis factor- $\alpha$, suggesting that the fibroblast model is predictive of the animal response.

Diagnosing intramammary infections: Comparison of multiple versus single quarter milk samples for the identification of intramammary infections in lactating dairy cows. By Dohoo et al., page 5515. Duplicate quarter milk samples were collected daily for 5 consecutive days with the infection status of the quarter determined by culture results from $\mathrm{d} 1,3$, and 5 . Various combinations of the samples from d 2 and 4 were evaluated for their ability to predict the infection status. Requiring 2 positive samples resulted in very few false positives but many false negatives. If a sample was considered positive and it was positive on either of 2 samples, there were fewer false negatives but more false positives. Overall, triplicate sampling did the best job of minimizing false positives and false negatives.

Effects of prepartum 2,4-thiazolidinedione on insulin sensitivity, plasma concentrations of tumor necrosis factor- $\alpha$ and leptin, and adipose tissue gene expression. By Schoenberg et al., page 5523. An insulin sensitizing agent was used in dairy cattle for $3 \mathrm{wk}$ prior to calving to improve metabolic health during the transition period. In addition to improving intake and decreasing circulating nonesterified fatty acids around the time of calving, the agent increased peroxisome profilerator-activated receptor $\gamma$ mRNA in adipose tissue and increased circulating tumor necrosis factor- $\alpha$. This study suggests alternative mechanisms for the positive effects of prepartum administration of an insulin-sensitizing agent and illustrates temporal changes in plasma leptin and tumor necrosis factor- $\alpha$ around the time of calving.

Short communication: Long-term survival of flag ear-tags on an Israeli dairy farm. By Seroussi et al., page 5533. Although 2-plated self-piercing eartags were developed in the 19th century, little information is available in the scientific literature on their retention rates. A method is presented that facilitates estimation of eartag retention rate and shows that the yearly retention rate of flag eartags was lower than expected (0.9) on the Israeli dairy farm based on a long-term $(>3 \mathrm{yr}$ ) field test. Tag design and on-farm management were factors affecting tag retention. 
The influence of colostral immunoglobulin concentration in heifer calves' serum on their health and growth. By Furman-Fratczak et al., page 5536. The aim of this study was to determine factors affecting passive transfer in dairy heifer calves and how it influenced rearing results up to first insemination. Calves were divided into 4 groups based on serum immunoglobulin concentration at 30 to $60 \mathrm{~h}$ of life (group 1: $<5 \mathrm{~g} / \mathrm{L}$; group 2: 5 to $10 \mathrm{~g} / \mathrm{L}$; group 3: 10 to $15 \mathrm{~g} / \mathrm{L}$; group 4: $>15 \mathrm{~g} / \mathrm{L})$. Poor vitality of calves associated with dystocia that occurred more frequently in primiparous cows and the origin of these calves negatively influenced passive transfer. Heifers from group 4 did not succumb to pneumonia and were inseminated $30 \mathrm{~d}$ earlier than those of group 1.

Effects of dietary protein concentration and coconut oil supplementation on nitrogen utilization and production in dairy cows. By Lee et al., page 5544. A reduced crude protein diet (14.8\%) depressed fiber digestibility and decreased milk production compared with a control crude protein (16.7\%) diet. The low-protein diet reduced urinary and total nitrogen losses and increased milk nitrogen efficiency. Supplementing the low-protein diet with coconut oil decreased ruminal protozoal counts, but depressed feed intake and consequently, cow productivity. Coconut oil also caused milk fat depression. Despite the reduced protozoal counts, coconut oil supplementation had no effect on the population of methanogens in the rumen.

Effect of amino acid or casein supply on wholebody, splanchnic, and mammary glucose kinetics in lactating dairy cows. By Galindo et al., page 5558. Protein and energy metabolism interact: wholebody (WB) availability, measured as the rate of appearance (Ra), of glucose increases with amino acid supply in dairy cows. In this study, amino acid supply increased glucose WB Ra up to $24 \%$ : the liver was responsible for this augmentation. Overall, liver synthesis and true portal absorption of glucose accounted for 78 and $21 \%$ of glucose WB Ra, respectively. The majority of this glucose, $73 \%$, was used by the mammary gland, of which $73 \%$ was secreted as milk lactose. The interaction between energy and protein nutrients needs to be considered when balancing dairy rations.

Effects of essential oils on milk production and composition, and rumen microbiota in Chios dairy ewes. By Giannenas et al., page 5569. We investigated the effects of essential oil addition on the performance and rumen parameters of a high-yielding Greek dairy sheep breed. We show that addition of essential oils improved milk yield and feed utilization in a dosedependent manner, while having small effects on milk composition. At the same time, addition of essential oils affected the composition of rumen microbial populations and fermentation parameters. Although not all mechanisms by which the performance improvements were achieved are well understood, their consequences would be of significance for dairy sheep systems.

Effect of method of delivery of sodium butyrate on rumen development in newborn calves. By Górka et al., page 5578. Justified for economic reasons, early weaning of dairy calves requires rapid rumen development, which may be stimulated by dietary supplementation of sodium butyrate. However, the optimal delivery method for sodium butyrate supplementation (milk replacer or starter mixture) has not been determined for dairy calves. Addition of sodium butyrate in the starter mixture directly stimulated rumen development as determined by higher reticulorumen weight and better developed rumen papillae, whereas sodium butyrate in milk replacer stimulated rumen development indirectly, probably by a positive effect on performance and health of calves.

Effect of Lactobacillus buchneri LN4637 and Lactobacillus buchneri LN40177 on the aerobic stability, fermentation products, and microbial populations of corn silage under farm conditions. By Tabacco et al., page 5589. The economic damage that results from aerobic deterioration is a significant problem for farm profitability and feed quality throughout the world. Inoculants containing Lactobacillus buchneri on its own or in combination with homofermentative lactic acid bacteria (Lactobacillus casei) applied to corn silage stored in commercial farm bunker silos at a rate of $1 \times 10^{5}$ cfu of L. buchneri per gram of herbage, could be effective in enhancing the aerobic stability of silage by directing silage fermentation towards a more heterolactic type of fermentation, reducing the amount of yeast, and thus increasing the time that silage could remain stable upon exposure to air.

Transfer of near-infrared spectrometric models for silage crude protein detection between different instruments. By Liu et al., page 5599. This study was undertaken to distinguish how collected near-infrared reflectance spectroscopy (NIRS) spectra of silages differ on different instruments, and how well the different techniques for transferring NIRS calibrations perform for crude protein detection. Two Fourier transform instruments and 1 scanning grating instrument were involved. The slope/bias (SB), local centering (LC), orthogonal signal correction (OSC), direct standardization, and piecewise direct standardization transfer methods were tested and evaluated. The spectra obtained with 3 instruments were obviously different. The SB, OSC, and LC techniques were successful for calibration transfer between 2 Fourier transform 
instruments, whereas the OSC method was moderately useful for calibration transfer between Fourier transform and scanning grating instruments.

Effectiveness of modified yeast cell wall extracts to reduce aflatoxin $\mathrm{B} 1$ absorption in dairy ewes. By Firmin et al., page 5611. Aflatoxins are toxic fungal metabolites associated with feedstuffs that can affect production and health of livestock and could affect consumers as toxins can be transferred into animal products. A modified yeast cell wall extract changed the excretion balance of aflatoxin-contaminated feed fed to dairy ewes. The observed increase in aflatoxin elimination in feces is a direct indication that the yeast product impaired aflatoxin absorption and suggests that dietary supplementation with this yeast cell wall extract could protect ruminants against the toxic effects of aflatoxins in feed.

Postabsorptive carbohydrate adaptations to heat stress and monensin supplementation in lactating Holstein cows. By Baumgard et al., page 5620. Study objectives were to evaluate metabolic adaptations in pair-fed thermal neutral control and heatstressed lactating cows. Data suggest that heat-stressed cows use a larger proportion of hepatic-produced glucose for nonproductive purposes. This results from an apparent inability to enlist glucose-sparing mechanisms, resulting in an increased contribution of glucose to whole-animal energetics. Consequently, glucose availability for mammary lactose synthesis is diminished; however, overall milk yield decreases $(\sim 50 \%)$ more than would be expected based on reductions in nutrient intake alone.

Starch and oil in the donor cow diet and starch in substrate differently affect the in vitro ruminal biohydrogenation of linoleic and linolenic acids. By Zened et al., page 5634. The intermediates of ruminal metabolism of polyunsaturated fatty acids can affect both cow milk production and human consumer's health, especially trans-10 isomers, which exhibit some negative effects. This study outlined that the trans-10 shift of linoleic acid biohydrogenation due to a high starch plus sunflower oil diet is associated with changes in bacterial community, and demonstrated that microbiota of cows exhibiting a trans-10 shift does not result in formation of trans-10 isomers when incubated with linolenic acid.

Plasma potassium-lowering effect of oral glucose, sodium bicarbonate, and the combination thereof in healthy neonatal dairy calves. By Grünberg et al., page 5646. Hyperkalemia is frequently encountered in neonatal calves with diarrhea. Although diarrheic calves are routinely treated with glucose and sodium bicarbonate to correct acid-base and energy imbalances, the effect of these compounds on the potassium homeostasis of calves has not yet been studied. This study reports a strong plasma potassium-lowering effect of sodium bicarbonate and glucose and provides an improved understanding of the mechanism of action of these compounds on potassium homeostasis in calves.

Short communication: Addition of varying amounts of sodium bicarbonate to colostrum replacer: Effects on immunoglobulin G absorption and serum bicarbonate in neonatal calves. $B y$ Cabral et al., page 5656. Previous studies identified sodium bicarbonate as an inexpensive colostrum replacer supplement that may increase absorption of immunoglobulins in calves. Our study evaluated graded doses of sodium bicarbonate added to colostrum replacer and the effects on immunoglobulin $\mathrm{G}$ absorption and serum bicarbonate concentrations. Immunoglobulin G concentration tended to be reduced and apparent efficiency of absorption of immunoglobulin $\mathrm{G}$ was reduced, whereas serum bicarbonate was increased by supplementing colostrum replacer with incremental amounts of sodium bicarbonate. Increased serum bicarbonate concentrations inhibit absorption of immunoglobulin $\mathrm{G}$ when supplementing colostrum replacer with $45 \mathrm{~g} /$ feeding of sodium bicarbonate.

Short communication: Effect of calf starter on rumen $\mathrm{pH}$ of Holstein dairy calves at weaning. By Laarman and Oba, page 5661. Calves are fed a readily fermentable calf starter prior to weaning to facilitate rumen development, but excess fermentation in the rumen often results in low $\mathrm{pH}$ and impairs rumen function. In this study, we found that rumen $\mathrm{pH}$ was not different between calves fed a calf starter and those not fed a calf starter, suggesting that calf starter consumption may not be the primary factor affecting rumen $\mathrm{pH}$. However, hay intake was negatively correlated to severity of rumen acidosis, suggesting that hay intake is important in reducing rumen acidosis.

Genetic analysis of milk urea nitrogen and relationships with yield and fertility across lactation. By Mucha and Strandberg, page 5665. This study focused on the relationship of milk urea nitrogen (MUN) with milk production and fertility. It aimed to enhance knowledge about factors influencing fertility, a trait of high economic importance. Test-day records of first-lactation Swedish Holstein cows were analyzed. A weak positive genetic relationship was found between MUN and fertility traits, which would imply that animals with high merit for MUN also have better fertility.

Genomic inbreeding and relationships among Holsteins, Jerseys, and Brown Swiss. By VanRaden et al., page 5673. Methods to combine ge- 
nomic and pedigree relationships were compared using genotypes of Holsteins, Jerseys, and Brown Swiss by estimating adjustments for means and regressions of genomic on pedigree relationships. Adjustments for base population allele frequencies and adjustments to make pedigree relationships match genomic relationships more closely in multi-breed populations were also determined. We found that genomic inbreeding accurately detected pedigree inbreeding and that breed identity can be determined more accurately using all markers than marker subsets. These results provide a basis for future multi-breed genomic evaluations.

Predicting bovine milk protein composition based on Fourier transform infrared spectra. By Rutten et al., page 5683. In this study, bovine milk protein composition was predicted based on milk mid-infrared spectra. We found that predictions of individual protein composition were too inaccurate to enable selection of individual animals. However, for specific purposes when, for example, groups of animals that meet a certain threshold are to be selected, the presented model could be useful in practice on the phenotypic level. Genetic correlations between electrophoresis-determined and mid-infrared-predicted protein composition traits showed that when used as input to estimate breeding values, observations of predicted protein composition provide an excellent means for genetic improvement of protein composition.

Short communication: Translational efficiency of Mediterranean river buffalo casein transcripts. By Cosenza et al., page 5691. Caseins $\left(\alpha_{S 1}, \beta, \alpha_{S 2}\right.$, and $\kappa)$ are the most abundant proteins in ruminant milk and their role in the cheese making process is well known. It is known that differences in their expression level influence the yield and technological and organoleptic characteristics of cheeses. Unlike the situation in cattle, sheep, and goats, no research has been carried out on the expression of casein genes in buffalo species and on their translational efficiency. In this study, we report that the 4 buffalo casein genes are not transcribed and translated with the same efficiency.

Short communication: Distribution of recessive genetic defect carriers in Chinese Holstein. By Sun et al., page 5695. In total, 732 proven bulls and
136 young bulls entering the national genetic improvement programs for dairy cattle of China were tested for complex vertebral malformation, bovine leukocyte adhesion deficiency, citrullinemia and deficiency of uridine monophosphate synthase, the most important recessive hereditary diseases in cattle. The proportions of their carriers were found to be $7.72,1.38,0.23$, and $0.12 \%$, respectively. Establishing a monitoring system on inherited disorders for proven and young bulls will prevent further spread of the defective alleles in Chinese dairy cattle.

Monitoring of sensory attributes used in the quality payment system of Trentingrana cheese. By Bittante et al., page 5699. Wheels of Trentingrana cheese were evaluated by 8 experts for 7 sensory attributes, and the scores were combined in a quality index used to establish price premiums or penalties for the cheese. The quality index was influenced more by flavor (odor, taste, and aroma) than by visually assessed traits (external aspect, rind thickness, paste color, and texture). Both quality index and sensory attributes were explained by dairy, year and season of production, and, with few exceptions, by first-order interactions between the main effects. Dairy was the most important source of variation for quality index and visually assessed traits, whereas year of production was the most important for flavor attributes.

Effects of stored feed cropping systems and farm size on the profitability of Maine organic dairy farm simulations. By Hoshide et al., page 5710. Northeast organic dairy farmers want to maximize onfarm production of protein and energy and minimize purchased inputs. Computer simulation of 3 organic feed systems showed perennial sod-based forage systems were profitable for small farms because of lower equipment and storage costs. Corn-based forage systems were more profitable for large farms, which raised row crops on a more efficient scale. For medium-sized farms, the corn-based system was more profitable when perennial forage harvest was delayed and resulted in compromised feed quality. Perennial sod-based systems were more competitive when purchased energy prices were relatively low and protein supplement prices were relatively high. 